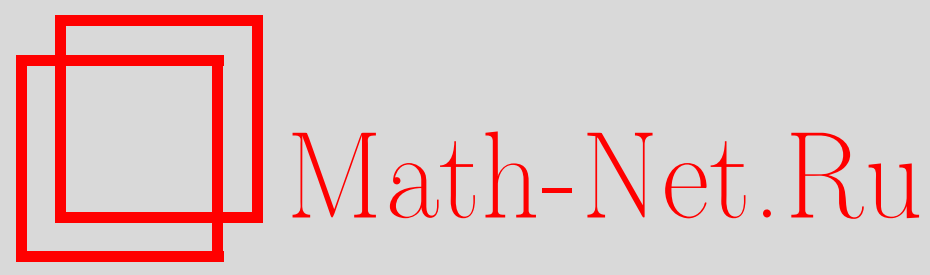

В. П. Ильев, Задачи на системах независимости, разрешимые жадным алгоритмом, Дискрет. матем., 2009, том 21, выпуск 4, 85-94

DOI: https://doi.org/10.4213/dm1074

Использование Общероссийского математического портала Math-Net.Ru подразумевает, что вы прочитали и согласны с пользовательским соглашением http://www . mathnet.ru/rus/agreement

Параметры загрузки:

IP: 34.227 .88 .159

26 апреля 2023 г., 09:46:40 
УДК 519.8

\title{
Задачи на системах независимости, разрешимые жадным алгоритмом
}

\author{
() 2009 г. В. П. Ильев
}

\begin{abstract}
Одним из центральных результатов теории матроидов является известная теорема Радо-Эдмондса, утверждающая, что задача максимизации аддитивной функции на системе независимости разрешима жадным алгоритмом тогда и только тогда, когда система независимости является матроидом.

Многочисленные обобщения теоремы Радо-Эдмондса в основном были связаны с расширением множества допустимых решений задачи максимизации, как правило, система независимости заменялась на систему достижимости. Расширение класса целевых функций также сопровождалось расширением допустимой области.

В настоящей работе исследуются задачи максимизации неаддитивных функций множеств на системах независимости. Предложена характеризация класса целевых функций задач на матроидах, разрешимых жадным алгоритмом. Доказано обобщение теоремы Радо-Эдмондса для задачи максимизации на системе независимости неаддитивной функции из указанного класса.
\end{abstract}

\section{1. Введение}

Объектом нашего исследования будут задачи максимизации вида

$$
\max \{f(X): X \in \mathscr{A}\}
$$

где $\mathscr{A}-$ непустое семейство подмножеств конечного множества $U$, а

$$
f: 2^{U} \rightarrow \mathbf{R}_{+}
$$

- монотонная неотрицательная функция множеств. Пару $\mathscr{S}=(U, \mathscr{A})$ будем называть системой множеств. Множества семейства $\mathscr{A}$ называются допустимыми.

Чаще всего в приложениях в качестве множества допустимых решений задачи (1) фигурируют системы независимости, обладающие тем свойством, что в них любое подмножество допустимого множества само допустимо. Как правило, в этих задачах требуется найти базу максимального веса, то есть максимальное по включению допустимое множество с максимальным значением целевой функции $f$.

Очень многие задачи комбинаторной оптимизации, сводящиеся к схеме (1) на системе независимости, являются $N P$-трудными. В качестве метода приближенного решения задачи (1) рассмотрим следующий простой алгоритм, являющийся дискретным аналогом алгоритма наискорейшего подъема. 
Жадный алгоритм (GA) . Итерация 0. $X \leftarrow \varnothing$. Перейти на итерацию 1 .

Итерация $k, k \geqslant 1$. Выбрать такой элемент $x_{k} \in U \backslash X$, что

$$
f\left(X \cup x_{k}\right)=\max _{\substack{x \in U \backslash X \\ X \cup x \in \mathscr{A}}} f(X \cup x),
$$

$X \leftarrow X \cup x_{k}$ и перейти на итерацию $k+1$. Если такого элемента $x_{k} \in U \backslash X$ нет, то $S_{\mathrm{GA}} \leftarrow X$.

Конец.

Здесь и всюду далее в обозначении одноэлементного множества $\{x\}$, как правило, будут опускаться фигурные скобки.

Один из центральных результатов теории матроидов - известная теорема РадоЭдмондса $[1,2]$ о том, что задача (1) на системе независимости разрешима жадным алгоритмом для любой аддитивной целевой функции тогда и только тогда, когда система независимости является матроидом.

Многочисленные обобщения теоремы Радо-Эдмондса в основном были связаны с расширением допустимой области. Как правило, множество допустимых решений задачи (1) заменялось на систему достижимости, в которой для любого непустого допустимого множества существует его допустимое подмножество, на единицу меньшей мощности. Примерами таких систем являются гридоиды и матроидные вложения, которые можно рассматривать как обобщения матроидов. Так, в работах [3, 4] охарактеризован класс гридоидов, на которых задача (1) разрешима жадным алгоритмом для любой аддитивной целевой функции, а в [5] установлены аналогичные критерии для более общих классов задач. В [6] доказано, что задача (1) на системе достижимости разрешима жадным алгоритмом для любой аддитивной целевой функции тогда и только тогда, когда допустимая область является матроидным вложением.

Обобщения теоремы Радо-Эдмондса за счет расширения класса целевых функций также сопровождались расширением допустимой области. В работе [7] доказано обобщение теоремы Радо-Эдмондса для задачи максимизации вогнутой сепарабельной функции на системе целочисленных векторов, обладающей свойством наследственности. Этот результат получил дальнейшее развитие в работе [8]. В [9] исследованы задачи на гридоидах с нелинейными (неаддитивными) целевыми функциями, а в [6] описан класс целевых функций задач на матроидных вложениях, разрешимых жадным алгоритмом.

Мы сосредоточим усилия на изучении задач вида (1) на системах независимости. Это сделано по двум причинам. Во-первых, системы независимости представляют собой классический объект, имеющий многочисленные приложения. Во-вторых, как будет показано далее, любая задача (1), в которой требуется найти базу произвольной системы множеств, эквивалентна задаче на системе независимости.

В данной работе доказано обобщение теоремы Радо-Эдмондса для задачи (1) на системе независимости с неаддитивной целевой функцией и предложена характеризация класса целевых функций задач на матроидах, разрешимых жадным алгоритмом. 


\section{2. Определения и известные результаты}

Рассмотрим задачу максимизации в следующей постановке:

$$
\max \{f(X): X \in \mathscr{B}\},
$$

где $\mathscr{B}$ - семейство всех баз, то есть максимальных по включению подмножеств системы множеств $\mathscr{Y}=(U, \mathcal{A})$, а $f: 2^{U} \rightarrow \mathbf{R}_{+}-$монотонная неотрицательная функция множеств.

Система множеств $\mathscr{S}=(U, \mathscr{A})$ называется системой независимости или наследственной системой, если семейство $\mathscr{A}$ непусто и удовлетворяет следующей аксиоме наследственности:

$$
\text { если } A \in \mathscr{A} \text { и } A^{\prime} \subseteq A \text {, то } A^{\prime} \in \mathscr{A} \text {. }
$$

Множества семейства $\mathscr{A}$ называются независимыми.

Замечание 1. Жадный алгоритм всегда находит базу системы независимости, то есть допустимое решение задачи (2) на системе независимости.

Замечание 2. Задача (2) на произвольной системе множеств $\mathscr{S}=(U, \mathscr{A})$ эквивалентна некоторой задаче максимизации на системе независимости.

Действительно, в задаче (2) на произвольной системе множеств $\mathscr{S}=(U, \mathscr{A})$ мы можем заменить систему $\mathscr{S}$ на ее наследственное замыкание $\mathscr{S}^{*}=\left(U, \mathscr{A}^{*}\right)$, где

$$
\mathscr{A}^{*}=\left\{A^{*} \subseteq A \mid A \in \mathscr{A}\right\} .
$$

Понятно, что $\mathscr{S}^{*}$ - система независимости, и множество оптимальных решений задачи (2) на системе $\mathscr{S}$ совпадает с множеством оптимальных решений аналогичной задачи на системе $\mathscr{S}^{*}$.

Система независимости $\mathscr{S}=(U, \mathscr{A})$ называется матроидом, если семейство $\mathscr{B}$ всех ее баз обладает следующим свойством:

$$
\begin{aligned}
\text { если } B_{1}, B_{2} \in \mathscr{B} \text { и } b_{1} \in B_{1} \backslash B_{2}, \\
\\
\text { то } \exists b_{2} \in B_{2} \backslash B_{1} \text { такой, что }\left(B_{2} \cup b_{1}\right) \backslash b_{2} \in \mathscr{B} .
\end{aligned}
$$

Следующая теорема Радо-Эдмондса является одной из центральных в теории матроидов.

Теорема $1([1,2])$. Пусть $\mathscr{S}=(U, \mathcal{A})$ - система независимости. Жадный алгоритм находит оптимальное решение задачи (2) для любой аддитивной функции $f: 2^{U} \rightarrow \mathbf{R}_{+}$ тогда и только тогда, когда $\mathscr{S}$ - матроид.

Если целевая функция задачи (2) на матроиде не является аддитивной, то жадный алгоритм может не найти оптимальное решение. Однако существуют задачи на матроидах с неаддитивными целевыми функциями, в которых жадный алгоритм гарантированно находит оптимальное решение.

Пример 1. Рассмотрим следующую функцию, определенную на подмножествах множества $U=\left\{x_{1}, x_{2}, x_{3}\right\}$ :

$$
\begin{gathered}
f(\varnothing)=0, \quad f\left(\left\{x_{1}\right\}\right)=5, \quad f\left(\left\{x_{2}\right\}\right)=4, \quad f\left(\left\{x_{3}\right\}\right)=6, \\
f\left(\left\{x_{1}, x_{2}\right\}\right)=f\left(\left\{x_{1}, x_{3}\right\}\right)=9, \quad f\left(\left\{x_{2}, x_{3}\right\}\right)=10, \quad f(U)=11 .
\end{gathered}
$$

Функция множеств в этом примере неаддитивна, но, как нетрудно проверить, жадный алгоритм находит оптимальное решение задачи максимизации на любом матроиде $\mathscr{S}=(U, \mathscr{A})$. 
Наша цель - получить обобщение теоремы Радо-Эдмондса для задачи (2) на системе независимости с неаддитивной целевой функцией и охарактеризовать класс целевых функций задач (2) на матроидах, разрешимых жадным алгоритмом.

В работе [6] подобные результаты доказаны для задач на системах достижимости и матроидных вложениях. Сформулируем их.

Система множеств $\mathscr{S}=(U, \mathscr{A})$ называется системой достижимых множеств или системой достижимости, если семейство $\mathscr{A}$ непусто и удовлетворяет следующей аксиоме:

$$
\text { если } A \in \mathscr{A} \text { и } A \neq \varnothing \text {, то } \exists a \in A \text {, такой, что } A \backslash a \in \mathscr{A} \text {. }
$$

Система достижимости $\mathscr{S}=(U, \mathscr{A})$ с семейством баз $\mathscr{B}$ называется расширяемой, если она обладает следующим свойством:

$$
\text { если } A \in \mathscr{A}, B \in \mathscr{B} \text { и } A \subset B \text {, то } \exists b \in B \backslash A \text {, такой, что } A \cup b \in \mathscr{A} \text {. }
$$

Система достижимости $\mathscr{S}=(U, \mathscr{A})$ называется конгруэнтно-замкнутой, если она удовлетворяет следующей аксиоме:

$$
\begin{array}{r}
\forall A \in \mathcal{A}, \forall u, v \in \operatorname{ext}(A), \forall V \subseteq U \backslash A \backslash \operatorname{ext}(A) \\
\text { из } A \cup u \cup V \in A^{*} \text { следует, что } A \cup v \cup V \in A^{*},
\end{array}
$$

где

$$
\operatorname{ext} A=\{u \in U \mid A \cup u \in \mathscr{A}\}
$$

$\mathscr{S}^{*}=\left(U, \mathscr{A}^{*}\right)-$ наследственное замыкание системы $\mathscr{Y}$.

Матроидное вложение - это система достижимости, которая является расширяемой, конгруэнтно-замкнутой и наследственное замыкание которой есть матроид. Очевидно, что матроидное вложение, являющееся системой независимости, совпадает с матроидом.

В статье [6] доказано следующее обобщение теоремы Радо-Эдмондса.

Теорема 2 ([6]). Пусть $\mathscr{S}=(U, A)-$ система достижимости. Жадный алгоритм находит оптимальное решение задачи (2) на системе 9 для любой аддитивной функиии $f: 2^{U} \rightarrow \mathbf{R}_{+}$тогда и только тогда, когда $\mathscr{S}$ - матроидное вложение.

Как и в случае задач на матроидах, существуют задачи на матроидных вложениях с неаддитивными целевыми функциями, в которых жадный алгоритм гарантированно находит оптимальное решение. В [6] получена характеризация целевых функций задач на матроидных вложениях, разрешимых жадным алгоритмом. Чтобы сформулировать этот результат, нам потребуется следующее определение.

Определение 1. Монотонная функция множеств $f: 2^{U} \rightarrow \mathbf{R}_{+}$называется согласованной, если для любых множеств $T \subset T^{\prime} \subset U$ и для любых элементов $x, y \in U \backslash T^{\prime}$ таких, что

$$
f(T \cup x) \geqslant f(T \cup y),
$$

имеет место неравенство

$$
f\left(T^{\prime} \cup x\right) \geqslant f\left(T^{\prime} \cup y\right) .
$$

Очевидно, что любая аддитивная функция является согласованной. 
Теорема 3 ([6]). Пусть $f: 2^{U} \rightarrow \mathbf{R}_{+}-$неотрицательная функиия множеств. Жадный алгоритм находит оптимальное ремение задачи (2) с иелевой функиией $f$ на любом матроидном вложении $\mathscr{S}=(U, \mathscr{A})$ тогда и только тогда, когда функиия $f$ является согласованной.

Далее в разделе 4 будет доказана аналогичная теорема для матроидов.

\section{3. Градиентно-согласованные функции}

Чтобы доказать критерий разрешимости задачи (2) на матроиде, нам придется несколько расширить класс согласованных функций.

Пусть $U-$ конечное множество, $f: 2^{U} \rightarrow \mathbf{R}_{+}$. Рассмотрим множество $V \subseteq U$ мощности $|V| \geqslant 3$, и пусть натуральное число

$$
k \leqslant|V|-2 .
$$

Множество $X \subset V$ будем называть градиентным подмножеством множества $V$, если существует такое упорядочение $X=\left\{x_{1}, \ldots, x_{k}\right\}$ его элементов, что

$$
\begin{array}{rlrl}
f\left(\left\{x_{1}\right\}\right) & \geqslant f(\{x\}) & & \text { для любого } x \in V, \\
f\left(\left\{x_{1}, x_{2}\right\}\right) & \geqslant f\left(\left\{x_{1}, x\right\}\right) & & \text { для любого } x \in V \backslash x_{1}, \\
& \ldots & & \\
f(X) & \geqslant f\left(\left\{x_{1}, \ldots, x_{k-1}, x\right\}\right) & \text { для любого } x \in V \backslash\left\{x_{1}, \ldots, x_{k-1}\right\} .
\end{array}
$$

В дальнейшем, если $X$ - градиентное подмножество множества $V$, то в записи $X=\left\{x_{1}, \ldots, x_{k}\right\}$ будет указываться именно тот порядок элементов, о котором идет речь в определении.

Монотонная функция множеств $f: 2^{U} \rightarrow \mathbf{R}_{+}$называется градиентно-согласованной, если для любых множеств $T \subset T^{\prime} \subset U$ и для любых элементов $x, y \in U \backslash T^{\prime}$ таких, что множество $T \cup x=\left\{x_{1}, \ldots, x_{k-1}, x\right\}$ является градиентным подмножеством множества $T^{\prime} \cup\{x, y\}$, справедливо неравенство (6).

Заметим, что это определение отличается от определения согласованной функции тем, что выполнение неравенства (6) требуется теперь не для любых множеств $T \subset T^{\prime} \subset U$ и любых элементов $x, y \in U \backslash T^{\prime}$, а лишь для таких, что $T \cup x$ является градиентным подмножеством множества $T^{\prime} \cup\{x, y\}$. При этом, как легко видеть,

$$
f(T \cup x) \geqslant f(T \cup z), \quad z \in\left(T^{\prime} \cup\{x, y\}\right) \backslash T,
$$

так что требование

$$
f(T \cup x) \geqslant f(T \cup y)
$$

становится излишним. Отсюда вытекает следующее утверждение.

Замечание 3. Любая согласованная функция является градиентно-согласованной. В частности, любая аддитивная функция градиентно-согласована.

Однако, не всякая градиентно-согласованная функция является согласованной. Функция множеств $f$, описанная в примере 1 , не является согласованной: если

$$
T=\varnothing, \quad T^{\prime}=\left\{x_{3}\right\}, \quad x=x_{1}, \quad y=x_{2},
$$


Tо

$$
f(T \cup x)=f\left(\left\{x_{1}\right\}\right)>f\left(\left\{x_{2}\right\}\right)=f(T \cup y),
$$

но

$$
f\left(T^{\prime} \cup x\right)=f\left(\left\{x_{1}, x_{3}\right\}\right)<f\left(\left\{x_{2}, x_{3}\right\}\right)=f\left(T^{\prime} \cup y\right) .
$$

Однако, множество

$$
T \cup x=\left\{x_{1}\right\}
$$

не является градиентным подмножеством множества

$$
T^{\prime} \cup\{x, y\}=\left\{x_{1}, x_{2}, x_{3}\right\}=U .
$$

Поскольку это единственный случай невыполнения определения согласованной функции, функция $f$ является градиентно-согласованной.

Нам потребуется следующий критерий градиентно-согласованности функции.

Лемма 1. Монотонная функияия множеств $f: 2^{U} \rightarrow \mathbf{R}_{+}$является градиентно-согласованной тогда и только тогда, когда для любого множества $V \subseteq U$ мощности $|V| \geqslant 3 u$ любого его градиентного подмножества $X=\left\{x_{1}, \ldots, x_{k}\right\} \subset V$ справедливы неравенства

$$
f(V \backslash v) \geqslant f\left(V \backslash x_{k}\right)
$$

для всех $v \in V \backslash X$.

Доказательство. Докажем необходимость. Пусть функция $f$ является градиентно-согласованной. Рассмотрим произвольное множество $V \subseteq U$ мощности $|V| \geqslant 3$ и его градиентное подмножество

$$
X=\left\{x_{1}, \ldots, x_{k}\right\} \subset V .
$$

Пусть

$$
V=X \cup Y,
$$

где $|Y| \geqslant 2$.

Положим

$$
T=X \backslash x_{k}, \quad T^{\prime}=T \cup Y \backslash y,
$$

где $y-$ произвольный элемент множества $Y$. Поскольку

$$
T \cup x_{k}=X
$$

является градиентным подмножеством множества

$$
V=T^{\prime} \cup\left\{x_{k}, y\right\},
$$

справедливо соотношение

$$
f\left(T \cup x_{k}\right) \geqslant f(T \cup y) .
$$

Отсюда в силу (6) получаем, что

$$
f(V \backslash y)=f\left(T^{\prime} \cup x_{k}\right) \geqslant f\left(T^{\prime} \cup y\right)=f\left(V \backslash x_{k}\right),
$$


то есть

$$
f(V \backslash v) \geqslant f\left(V \backslash x_{k}\right)
$$

для всех $v \in V \backslash X$.

Необходимость доказана.

Докажем достаточность. Рассмотрим произвольные множества $T \subset T^{\prime} \subset U$ и любые элементы $x, y \in U \backslash T^{\prime}$ такие, что

$$
T \cup x=\left\{x_{1}, \ldots, x_{k-1}, x\right\}
$$

является градиентным подмножеством множества $T^{\prime} \cup\{x, y\}$. Положим

$$
V=T^{\prime} \cup\{x, y\}, \quad X=T \cup x,
$$

и пусть $x_{k}=x$. Тогда из (7) следует, что

$$
f\left(T^{\prime} \cup x\right)=f(V \backslash y) \geqslant f\left(V \backslash x_{k}\right)=f\left(T^{\prime} \cup y\right),
$$

то есть функция $f$ является градиентно-согласованной.

Лемма доказана.

\section{4. Задачи, разрешимые жадным алгоритмом}

В этом параграфе будет доказано обобщение теоремы Радо-Эдмондса для задачи (2) на системе независимости с неаддитивной целевой функцией и будет охарактеризован класс целевых функций задач на матроидах, разрешимых жадным алгоритмом.

Лемма 2. Если в задаче (2) $\mathscr{B}$ - семейство баз матроида $\mathscr{S}=(U, \mathcal{A})$, а иелевая функция $f: 2^{U} \rightarrow \mathbf{R}_{+}$является градиентно-согласованной, то жадньий алгоритм находит оптимальное решение задачи (2).

Доказательство. Пусть $\mathscr{S}=(U, \mathscr{A})$ - матроид, а целевая функция задачи (2) является градиентно-согласованной. Пусть жадным алгоритмом GA построена база $S_{\mathrm{GA}}=\left\{x_{1}, \ldots, x_{r}\right\}$ матроида $\mathscr{T}$.

Заметим, что если существует такой элемент $u \in U$, что

$$
f(\{u\})>f\left(\left\{x_{1}\right\}\right)
$$

то $u-$ петля матроида $\mathscr{Y}$, то есть $\{u\} \notin \mathscr{A}$. В дальнейшем элемент $u$ можно исключить из рассмотрения, так как все множества, содержащие $u$, будут зависимыми множествами матроида $\mathscr{Y}$. Поэтому сразу без ограничения общности будем считать, что матроид $\mathscr{S}$ не содержит петель.

Лемма будет доказана, если будет доказано, что

$$
f\left(S_{\mathrm{GA}}\right) \geqslant f(B)
$$

для любой базы $B$ матроида $\mathscr{S}$. 
Доказательство утверждения (8) проведем индукцией по

$$
\left|S_{\mathrm{GA}} \backslash B\right|=\left|B \backslash S_{\mathrm{GA}}\right| \text {. }
$$

Если $\left|S_{\mathrm{GA}} \backslash B\right|=1$, то

$$
S_{\mathrm{GA}} \backslash B=\left\{x_{k}\right\}, \quad k \in\{1, \ldots, r\},
$$

a

$$
B \backslash S_{\mathrm{GA}}=\{v\}
$$

для некоторого $v \in U \backslash S_{\mathrm{GA}}$. Если $k=r$, то в силу выбора алгоритма GA

$$
f\left(S_{\mathrm{GA}}\right)=f\left(\left\{x_{1}, \ldots, x_{r-1}, x_{r}\right\}\right) \geqslant f\left(\left\{x_{1}, \ldots, x_{r-1}, v\right\}\right)=f(B) .
$$

Если же $k<r$, то для множества

$$
V=B \cup x_{k}
$$

и его градиентного подмножества $X=\left\{x_{1}, \ldots, x_{k}\right\}$, согласно (7),

$$
f\left(S_{\mathrm{GA}}\right)=f(V \backslash v) \geqslant f\left(V \backslash x_{k}\right)=f(B) .
$$

Основание индукции установлено.

Предположим, что утверждение (8) верно для любой базы $B \subset U$, для которой

$$
\left|S_{\mathrm{GA}} \backslash B\right| \leqslant m-1, \quad m \geqslant 2 .
$$

Рассмотрим такую базу $B \subset U$ матроида $\mathscr{S}$, что

$$
\left|S_{\mathrm{GA}} \backslash B\right|=m \text {. }
$$

Пусть $k$ - наименьший номер такой, что $x_{k} \in S_{\mathrm{GA}} \backslash B, k \in\{1, \ldots, r\}$. Тогда для множества

$$
V=B \cup x_{k}
$$

и его градиентного подмножества

$$
X=\left\{x_{1}, \ldots, x_{k}\right\} \subset V,
$$

согласно (7),

$$
f(V \backslash v) \geqslant f\left(V \backslash x_{k}\right)=f(B)
$$

для всех $v \in V \backslash X$.

По аксиоме (4) для $x_{k} \in S_{\mathrm{GA}} \backslash B$ существует такой элемент $v \in B \backslash S_{\mathrm{GA}} \subset V \backslash X$, что множество

$$
B^{\prime}=\left(B \cup x_{k}\right) \backslash v=V \backslash v
$$

является базой матроида $\mathscr{~ . ~ Т а к и м ~ о б р а з о м , ~}$

$$
f\left(B^{\prime}\right) \geqslant f(B)
$$


в силу (9). А так как

$$
\left|S_{\mathrm{GA}} \backslash B^{\prime}\right|=\left|S_{\mathrm{GA}} \backslash B\right|-1=m-1,
$$

по предположению индукции справедливо неравенство

$$
f\left(S_{\mathrm{GA}}\right) \geqslant f\left(B^{\prime}\right) .
$$

Окончательно получаем, что

$$
f\left(S_{\mathrm{GA}}\right) \geqslant f\left(B^{\prime}\right) \geqslant f(B) .
$$

Утверждение (8), а вместе с ним и лемма 2 доказаны.

Из теоремы 1 и леммы 2 вытекает следующее обобщение теоремы Радо-Эдмондса для задачи максимизации на системе независимости.

Теорема 4. Пусть $\mathscr{S}=(U, \mathscr{A})-$ система независимости. Жадньй алгоритм находит оптимальное решение задачи (2) на системе $\mathscr{S}$ для любой градиентно-согласованной функции множеств $f: 2^{U} \rightarrow \mathbf{R}_{+}$тогда и только тогда, когда $\mathscr{S}$-матроид.

Доказательство. Докажем необходимость. Если $\mathscr{S}$ - не матроид, то по теореме РадоЭдмондса существует такая аддитивная, а значит, градиентно-согласованная функция $f$, что жадный алгоритм может не найти оптимальное решение задачи (2) с целевой функцией $f$.

Достаточность условия теоремы 4 следует из леммы 2.

Справедлив следующий критерий разрешимости жадным алгоритмом задачи (2) на матроиде.

Теорема 5. Пусть $f: 2^{U} \rightarrow \mathbf{R}_{+}-$неотрицательная функция множеств. Жадный алгоритм находит оптимальное решение задачи (2) с иелевой функцией $f$ на произвольном матроиде $\mathscr{S}=(U, \mathscr{A})$ тогда и только тогда, когда функиия $f$ является градиентно-согласованной.

Доказательство. Докажем необходимость. Предположим, что функция $f$ не является градиентно-согласованной. Докажем, что в этом случае существует матроид $\mathscr{T}=(U, \mathscr{A})$, на котором жадный алгоритм может не найти оптимальное решение задачи (2).

Поскольку функция $f$ не является градиентно-согласованной, по лемме 1 существуют такое множество $V \subseteq U$ мощности $|V| \geqslant 3$ и такое его градиентное подмножество $X=\left\{x_{1}, \ldots, x_{k}\right\} \subset V$, что

$$
f(V \backslash v)<f\left(V \backslash x_{k}\right)
$$

для некоторого $v \in V \backslash X$. Рассмотрим матроид $\mathscr{S}$ на множестве $U$, в котором имеется всего две базы

$$
\begin{aligned}
& B_{1}=V \backslash x_{k}, \\
& B_{2}=V \backslash v,
\end{aligned}
$$

а все элементы множества $U \backslash V$ являются петлями (то есть $\{u\} \notin \mathscr{A}$ для всех $u \in U \backslash V$ ).

Поскольку $X=\left\{x_{1}, \ldots, x_{k}\right\}$ является градиентным подмножеством множества $V$ и $X$ независимо (так как является подмножеством базы $B_{2}$ ), алгоритм GA на первых $k$ шагах может последовательно выбрать элементы $x_{1}, \ldots, x_{k}$. Это означает, что алгоритм 
построит базу $B_{2}$, содержащую элемент $x_{k}$, в то время как в силу (10) оптимальным решением задачи (2) на матроиде $\mathscr{Y}$ является база $B_{1}$, не содержащая $x_{k}$.

Необходимость доказана.

Докажем достаточность. Пусть функция множеств $f$ является градиентно-согласованной. Тогда в силу леммы 2 жадный алгоритм гарантированно найдет оптимальное решение задачи (2) с целевой функцией $f$ на любом матроиде $\mathscr{S}=(U, \mathscr{A})$.

Теорема доказана.

\section{Список литературы}

1. Rado R., Note on independence functions. Proc. London. Math. Soc. (1957) 7, №3, 300-320.

2. Edmonds J., Matroids and the greedy algorithm. Math. Programming (1971) 1, №2, 127-136.

3. Korte B., Lovász L., Greedoids and linear objective functions. SIAM J. Alg. Discrete Math. (1984) 5, №2, 229-238.

4. Goetschel R. H., Linear objective functions on certain classes of greedoids. Discrete Appl. Math. (1986) 14, №1, 11-16.

5. Шенмайер В. В., Максимизация линейной целевой функции с помощью жадного алгоритма. Дискретный анализ и исследование операџий, сер. 1 (1999) 6, №4, 104-120.

6. Helman P., Moret B. M. E., Shapiro N. D., An exact characterization of greedy structures. SIAM J. Discrete Math. (1993) 6, №2, 274-283.

7. Глебов Н. И., Об одном классе задач выпуклого целочисленного программирования. Управляемые системы (1973) 11, 38-42.

8. Глебов Н. И., Шенмайер В. В., О применимости алгоритма покоординатного подъема к задачам целочисленного программирования. Дискретный анализ и исследование операций, сер. 1 (2000) 7, №4, 38-47.

9. Korte B., Lovász L., Mathematical structures underlying greedy algorithm. Lect. Notes Comput. Sci. (1981) 117, 205-209.

Статья поступила 10.04.2007.

Переработанный вариант поступил 26.01.2009. 